\title{
Synthesis and Characterization of Hydroxyapatite Produced by Microwave Assisted Precipitation Technique
}

\begin{abstract}
Y.E. ŞIMŞEK ${ }^{a, *}$ AND Ş. AVCI ${ }^{b}$
${ }^{a}$,Bilecik Şeyh Edebali University, Chemical Engineering Department, Bilecik, Turkey

${ }^{b}$ Bilecik Şeyh Edebali University, Metallurgical and Materials Engineering Department, Bilecik, Turkey

In medical applications, the compliance and integration of bone with implant are a critical issue. Biocompatible metal substrates coated by suitable materials such as calcium phosphates, especially hydroxyapatite, exhibit excellent biocompatibility, high mechanical strength, good corrosion resistance and toughness. The use of these type of material coatings on the implant surfaces has recently drawn attention of many researchers. Many methods such as sol-gel and dip coating, electrochemical and electrophoretic deposition, plasma spraying, hot isostatic pressing and pulsed laser deposition have been employed for the production of hydroxyapatite artificially. Among these methods microwave-assisted precipitation technique in a simulated body fluid solution is the simplest and most efficient way to produce hydroxyapatite. Hydroxyapatite with a suitable molar ratio of $\mathrm{Ca} / \mathrm{P}$ is bioactive, biocompatible and osteoconductive, and enhances direct attachment of implant to the bone. In this study, hydroxyapatite powders were produced by microwave-assisted precipitation. The powder samples were analyzed in detail by SEM-EDX, XRD and FTIR. Analysis results showed that experimental parameters such as microwave irradiation power and exposure time had a considerable effect on $\mathrm{Ca} / \mathrm{P}$ molar ratios, surface morphologies and crystallinity of the powders.
\end{abstract}

DOI: 10.12693/APhysPolA.135.974

PACS/topics: biomaterial, hydroxyapatite, microwave assisted precipitation, characterization, crystallinity

\section{Introduction}

Tissue diseases and defects, especially bone and bonerelated diseases, are major problems which affect life quality and standard of living very seriously. To enhance the necessities of life the employment of bioceramics has caused a drastic change in the health sector over the fifty years. Bioceramics, a subset of ceramics, are highly biocompatible and antithrombogenic materials that fill defects in human bones and teeth enable bone grafting and fractures onto the bone and replace infected tissues [1]. Hydroxyapatite $(\mathrm{HA}), \mathrm{Ca}_{10}\left(\mathrm{PO}_{4}\right)_{6}(\mathrm{OH})_{2}$, is bioactive, biocompatible, and osteconductive. HA, due to its chemical and structural resemblance, bears a striking similarity to human bone [2]. The coating and/or deposition with HA of metal alloys assists the osseointegration of implants with tissues in contact and enhances direct attachments of implants to the bone [3]. To date, many methods such as sol-gel, dip coating, sputter coating, emulsion processing, spray pyrolysis, plasma spraying, pulsed laser, electrophoretic deposition, and microwave assisted precipitation and coating methods have been developed to produce HA artificially to perform HA coatings on metallic alloys and nonmetallic materials. These methods have in detail been reviewed and discussed by Asri et al. [4]. Among the aforementioned methods, the microwave assisted precipitation technique to produce HA comes to the fore because of a wide variety of marked

\footnotetext{
* corresponding author; e-mail: yunusemre.simsek@bilecik.edu.tr
}

advantages over the others such as microwave irradiation: (i) lowers the reaction time very considerably by maximizing energy transfer within the volume, (ii) enables reproducibility, (iii) yields small particle size and a narrow size distribution and, (iv) offers high yield and crystallinity [5].

In the present work, the authors produced different calcium hydroxyapatite samples by microwave-assisted chemical precipitation method in a simulated body fluid (SBF). The samples were allowed to precipitate at different microwave irradiation powers and exposure times. The surface morphology, crystallinity and molar ratio of the produced HA structures were characterized in detail by XRD, SEM-EDX, and FTIR. Also, their differences and resemblances to the bone-like calcium hydroxyapatite were assessed thoroughly.

\section{Experimental procedure}

To precipitate HA, $1000 \mathrm{~mL}$ stock solution of simulated body fluid (SBF) was prepared in accordance to the Kokubo protocol. The amount and order of reagents used in the experiments for the preparation of $1000 \mathrm{~mL}$ of SBF solution were elaborated by Kokubo and Tajadama [6]. This solution was precipitated in twelve different experimental conditions, i.e. microwave irradiation power $(300$, 450 , and $600 \mathrm{~W}$ ) and exposure time (1, 3, 6, and $9 \mathrm{~min})$. All the precipitates were designated as WWWT in which WWW refers to microwave irradiation power and $T$ to time. For example, the designated experimental code 4506 means $450 \mathrm{~W}$ of microwave irradiation power and 6 min of exposure time. As a microwave irradiation source, a household microwave oven (Samsung ME711K 
of $2450 \mathrm{MHz}, 1150 \mathrm{~W}, \mathrm{CM} 75 \mathrm{~S}$ Magnetron) was used in all the experiments. After microwave-assisted precipitation, all the precipitates were oven-dried at $40^{\circ} \mathrm{C}$ overnight and afterwards were calcinated in an atmosphere controlled oven at $900{ }^{\circ} \mathrm{C}$ for $2 \mathrm{~h}$.

Phase content and analysis of the precipitate were carried out by X-ray diffraction (XRD, PANanalytical X'Pert HT-XRD) with $\mathrm{CuK} \alpha$ radiation in the step size of $0.02^{\circ}$, at a scan speed of $1^{\circ} / \mathrm{min}$. HA crystallinity of the powder samples is calculated using the formula below [7].

$$
X_{C}=\left[1-\left(V_{112 / 300} / I_{300}\right)\right] \times 100 \%
$$

where $X_{C}$ is the crystallinity percentage of the produced HA samples. $I_{300}$ is the intensity of the (300) reflection peak, and $V_{112 / 300}$ intensity of the hollow between (112) and (300) reflection peaks. Fourier Transform Infrared Spectroscopy (FTIR Agilent Cary 630FTIR Spectrometer) was used to analyze functional groups in the samples and spectra were taken in the wavenumber of 500$4000 \mathrm{~cm}^{-1}$ with a resolution of $2 \mathrm{~cm}^{-1}$. The surface morphologies of the HA samples were obtained by Scanning Electron Microscope (SEM Zeiss Supra 40Vp Gemini), and elemental analysis was determined by energy dispersive spectrometer attached to the SEM.

\section{Results and discussion}

The XRD pattern, FTIR spectrum and SEM micrograph of the HA powder obtained only at the optimum conditions (the highest crystallinity percentage) are illustrated herein. The FTIR spectrum of the HA powder shown in Fig. 1a. is the typical peaks of $\mathrm{OH}^{-}, \mathrm{PO}_{4}^{3-}$, and $\mathrm{CO}_{3}^{2-}$ belonging to hydroxyapatite. The sharp peak at $3639 \mathrm{~cm}^{-1}$ can be attributed to $\mathrm{OH}^{-}$. The adsorptive bands of $\mathrm{PO}_{4}^{3-}$ are at $595 \mathrm{~cm}^{-1}$, $962 \mathrm{~cm}^{-1}$, and $1042 \mathrm{~cm}^{-1}$. The peaks with associated with $\mathrm{CO}_{3}^{2-}$ appear at $664 \mathrm{~cm}^{-1}, 885 \mathrm{~cm}^{-1}, 1412 \mathrm{~cm}^{-1}$, and $1469 \mathrm{~cm}^{-1}[2-5,7]$. XRD pattern of the HA sample is shown in Fig. 1b. The sharp peaks can be attributed to the presence of hydroxyapatite. The observed peaks match perfectly the standard diffraction pattern of $\mathrm{Ca}_{10}\left(\mathrm{PO}_{4}\right)_{6}(\mathrm{OH})_{2}$ (JCPDS Card No. 9-432).

The crystallinity of HA samples was calculated using Eq. (1). Crystallinity in the given experimental conditions is shown in Table I and Fig. 2. As shown in Ta-

ble I, using microwave irradiation led to increasing crystallinity. The governing step of calcium hydroxyapatite crystallization process is the diffusion-controlled step. It is clear that using microwave external source microwave irradiation power and exposure time affects the rate of atomic motion and brings down the activation energy. Hence, the atoms are rapidly transported to the lattice site and crystallinity is consequently increased [8]. Also, the results reveal that among the HA samples produced in the twelve experimental conditions the closest $\mathrm{Ca} / \mathrm{P}$ molar ratio to that of human bone and to that of theoretical HA was obtained at $450 \mathrm{~W}$ and $9 \mathrm{~min}$.

(a)

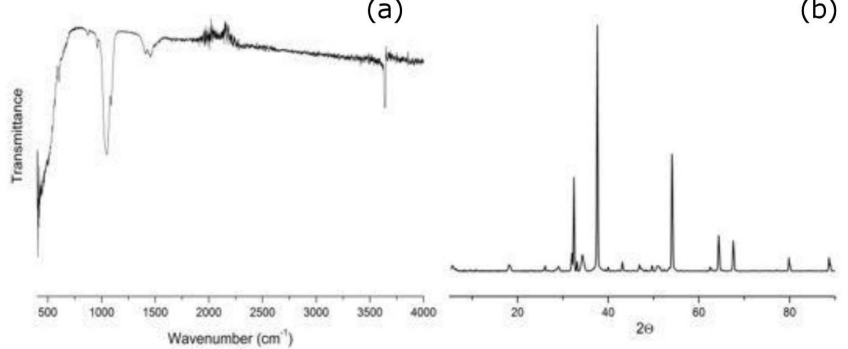

Fig. 1. FTIR spectrum (a) and X-ray diffraction pattern (b) of HA powder by precipitated by microwave irradiation power $(600 \mathrm{~W}, 9 \mathrm{~min})$.

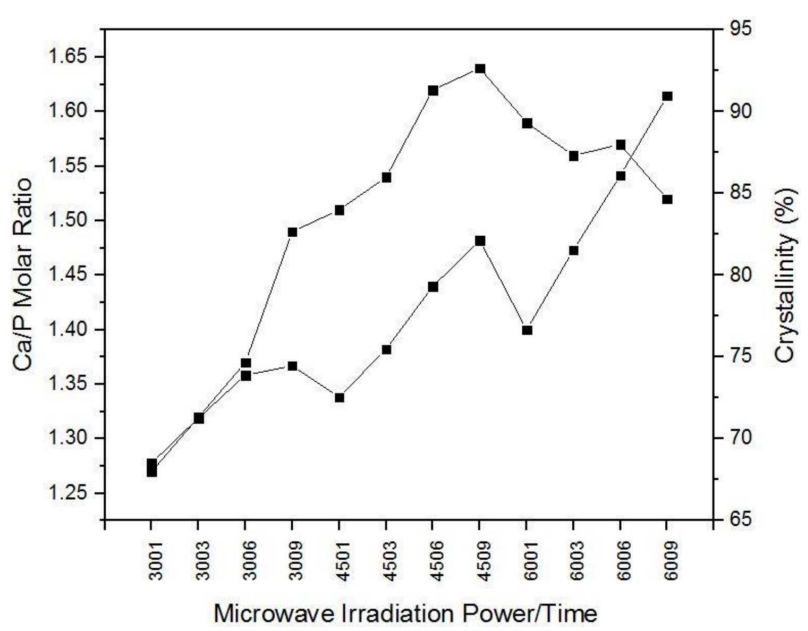

Fig. 2. The effect of microwave irradiation power and exposure time on the crystallinity and $\mathrm{Ca} / \mathrm{P}$ molar ratios.

Comparison of $\mathrm{Ca} / \mathrm{P}$ molar ratios, and crystallinity in twelve conditions. For comparision: for human bone

TABLE I $\mathrm{Ca} / \mathrm{P}$ molar ratio is $1.66[5,9]$.

\begin{tabular}{c|c|c|c|c|c}
\hline \hline \multicolumn{2}{c|}{ Precipitation conditions } & \multirow{2}{*}{ Crystallinity $X_{C}[\%]$} & \multicolumn{2}{|c|}{ Precipitation conditions } & \multirow{2}{*}{ Crystallinity $X_{C}[\%]$} \\
\cline { 4 - 5 } Designation & Ca/P (molar ratio) & & Designation & Ca/P (molar ratio) & \\
\hline 3001 & 1.27 & 68.54 & 4506 & 1.62 & 79.32 \\
3003 & 1.32 & 71.28 & 4509 & 1.64 & 82.15 \\
3006 & 1.37 & 73.89 & 6001 & 1.59 & 76.66 \\
3009 & 1.49 & 74.47 & 6003 & 1.56 & 81.56 \\
4501 & 1.51 & 72.56 & 6006 & 1.57 & 86.11 \\
4503 & 1.54 & 75.48 & 6009 & 1.52 & 90.98
\end{tabular}


The surface morphology of HA powder at different magnifications obtained at the optimum conditions is illustrated in Fig. 3a, b. It was observed that the produced HA powder at these conditions has sporadic morphology and is comprised of a cluster of micron-sized formless aggregates.

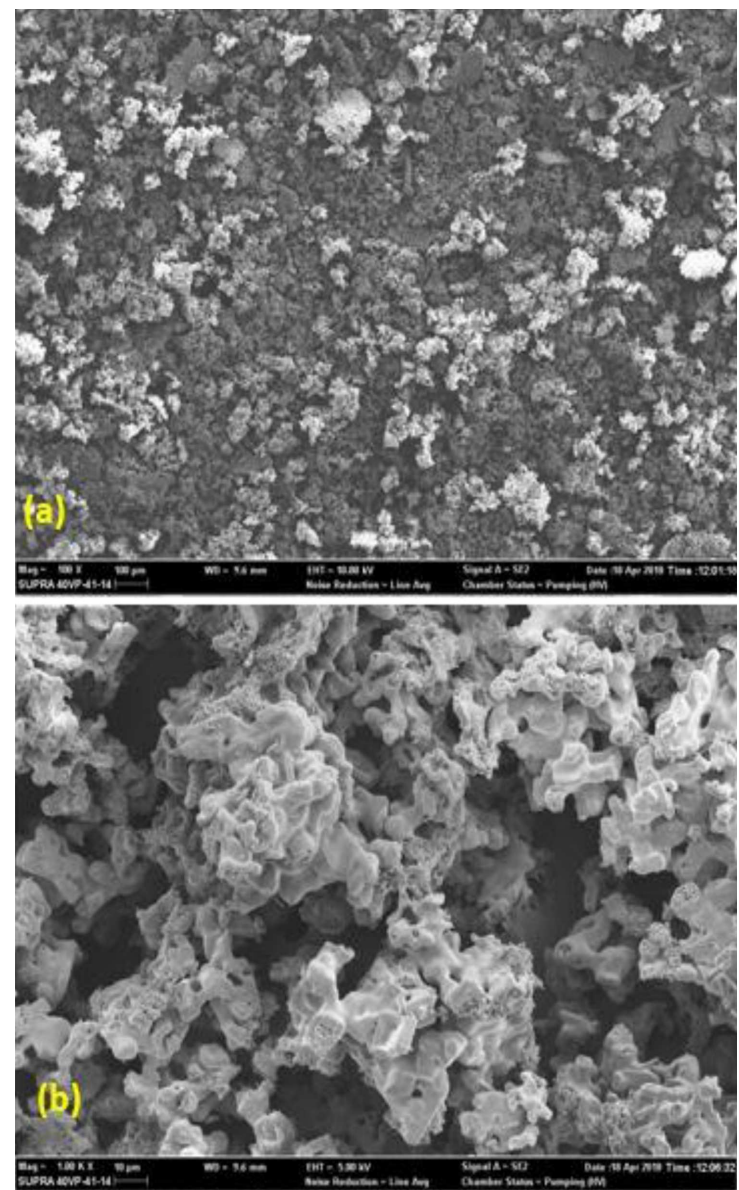

Fig. 3. SEM micrographs of HA powder by precipitated by microwave irradiation power $(600 \mathrm{~W}, 9 \mathrm{~min})$, Magnification (a) $100 \times$ (b) $1000 \times$.

\section{Conclusion}

In present work, hydroxyapatite with high crystallinity degree was successfully produced by microwave assisted precipitation method. The effects of the experimental parameters on the crystallinity and $\mathrm{Ca} / \mathrm{P}$ molar ratios of the HA powders produced were evaluated thoroughly. According to the results, the crystallinity of HA powders rose with increasing microwave irradiation power and exposure time, reaching a maximum at $600 \mathrm{~W}$ for $9 \mathrm{~min}$. The $\mathrm{Ca} / \mathrm{P}$ molar ratios varied from 1.27 to 1.64 irregularly. It was found in this study that owing to high crystallinity and $\mathrm{Ca} / \mathrm{P}$ molar ratio similar to human bone, microwave assisted precipitation method is a simple and efficient route for the production of HA.

\section{References}

[1] T. Yamamuro, Biomaterials Used in Orthopedics, in: Biomechanics and Biomaterials in Orthopedics Ed. D.G. Poitout, Springer, London 2004, p. 22.

[2] D. He, P, Liu, X. Liu, F. Ma, X. Chen, W. Li, J. Du, P. Wang, J. Zhao, J. Alloy. Compd. 672, 336 (2016).

[3] F. Zhang, S. Cai, G. Xu, F. Wang, N. Yu, R. Ling, X. Wu, Ceram. Int. 42, 18466 (2016).

[4] R. Asri, W. Harun, M. Hassan, S. Ghani, Z.Z. Buyong, J. Mech. Behav. Biomed. Mater. 57, 95 (2016).

[5] T.T. Demirtaş, G. Kaynak, M. Gümüşderelioğlu, Mater. Sci. Eng. C 49, 713 (2015).

[6] T. Kokubo, H. Takadama, Biomaterials 27, 2907 (2006).

[7] E. Landi, A. Tampieri, G. Celotti, J. Europ. Ceram. Soc. 20, 2377 (2000).

[8] A. Farzadi, M. Solati-Hashjin, F. Bakhshi, A. Aminian, Ceram. Int. 37, 65 (2011).

[9] X.Y. Zhao, Y.J. Zhu, F. Chen, B. Lu, J. Wu, Cryst. Eng. Comm. 15, 206 (2013). 